\author{
Petra Heißenberger \\ Pädagogische Hochschule Niederösterreich, Campus Baden \\ im Gespräch mit
}

Brigitte Schuckert

Bildungsdirektion für Niederösterreich, Sankt Pölten

\title{
Signals of Hope
}

DOI: https://doi.org/10.53349/sv.2021.i3.a119

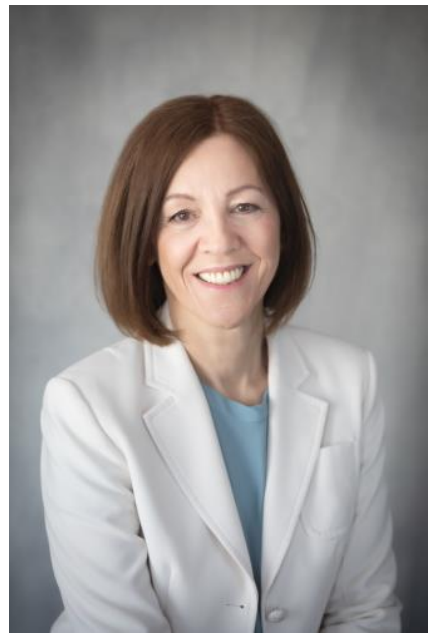

Foto: privat
Nach einigen Jahren in der Wirtschaft im In- und Ausland unterrichtete Brigitte Schuckert an der Berufsschule sowie an der Handelsakademie und Handelsschule. Berufliche Erfahrungen sammelte sie auch als Direktorin, als Landesschulinspektorin für kaufmännische Schulen sowie als Schulqualitätsmanagerin und Außenstellenleiterin der Bildungsregion 4 in Niederösterreich. Brigitte Schuckert ist Leiterin des Bereichs Pädagogischer Dienst in der Bildungsdirektion für Niederösterreich. Sie gestaltet seit vielen Jahren Schulentwicklung auf nationaler und internationaler Ebene aktiv mit und beteiligt sich immer wieder an Comenius- und Erasmus-Projekten. Die Absolvierung zahlreicher Lehrgänge im Bildungsbereich und außerschulischer Fortbildungsveranstaltungen im Finanzbereich sowie eine Business-Coaching-Ausbildung an der Freien Universität Berlin ermöglichen ihr, die gewonnenen Kenntnisse für die Schulen in Niederösterreich und in Zusammenarbeit mit dem BMBWF und der Landesregierung für Niederösterreich einzubringen.

Schule - der Ort des Vertrauens auf die Hoffnungsträger*innen der Zukunft! Welche Signale der Hoffnung kann/soll die Schule setzen und wie geschieht das im Unterricht? 
Sehr geehrte Leiterin des Bereiches Pädagogischer Dienst! Die letzte Frankfurter Buchmesse im Oktober 2020, Covid-bedingt erstmals online durchgeführt, stand unter dem Leitsatz „SIGNALS OF HOPE“. Welche Signale der Hoffnung soll, welche kann die Schule HEUTE setzen, um ihren Schüler*innen reale „Schule von morgen“ zu sein?

Brigitte Schuckert: Als „Signale der Hoffnung" wurden in Australien Leuchttürme bezeichnet, weil sie die ersten Gebäude waren, welche die aus Europa kommenden Segler*innen nach monatelanger Reise über das Meer sahen. Das Licht dieser Leuchttürme war für die einen ein Signal der neuen Heimat und für andere wiederum ein Signal für die Heimkehr. Nun könnte man dies auch mit der Schule von HEUTE in Verbindung setzen: Die Möglichkeiten der Unterrichtsgestaltung sollen Leuchttürme für die Schüler*innen werden, die sie anleiten, ihre Ziele erkennen und anzusteuern zu können. Signale der Hoffnung für unsere Schüler*innen sehe ich vor allem in den 21st Century Skills: Wir müssen unsere Schüler*innen zu kreativen, kritischen, kollaborierenden und kommunizierenden Menschen erziehen.

Bildungsgerechtigkeit ist gerade in Corona-Zeiten ein wieder viel beachtetes und viel beschriebenes Thema geworden, nicht selten wird es als politisches Argument genutzt. Was kann Schulleitung vor Ort konkret tun, um Bildungsungerechtigkeit zu minimieren, um die elterliche Hoffnung auf Gleichwertigkeit aller Schüler*innen zu nähren?

Bildungsungerechtigkeit sollte in unseren Schulen keinen Platz mehr finden. Träume der Schüler*innen über ihre berufliche und private Zukunft sollten mit der Unterstützung der Pädagog*innen für alle Schüler*innen erreichbar werden, egal welches Geschlecht die Schüler*innen haben, welche ethnische oder soziale Herkunft sie aufweisen.

\section{Persönliche Leuchttürme ansteuern}

Bildungsgerechtigkeit und somit Chancengerechtigkeit sollten unabhängig von religiöser oder politischer Anschauung möglich sein. Die Diversität der Gesellschaft hängt daher eng mit der Chancengerechtigkeit im Bildungsbereich zusammen, daher sollte jede Form der Ungerechtigkeit, vor allem im Bereich der Bildung, vermieden werden. Die Schüler*innen sollten somit ihre ganz persönlichen Leuchttürme ansteuern und erreichen können. Bildung ist dazu ein wesentlicher Schlüssel für die Schüler*innen, diese Leuchttürme anvisieren zu können.

Mit der Corona-Pandemie und dem damit einhergehenden digitalen Fernunterricht trat das Thema der Bildungsgerechtigkeit in die bildungspolitischen Diskussionen wieder verstärkt in den Vordergrund. Auch das Lernen in Beziehungen wurde dadurch in den Hintergrund gedrängt, was gerade Ungerechtigkeit ausgleichen könnte. Viele Schüler*innen konnten sich auch durch den Fernunterricht nicht in gleichem Maße beteiligen, da z. B. die technischen Voraussetzungen nicht gleich waren. Gerade in Zeiten von Krisen wird die Bildung in Mitleidenschaft gezogen. Daher müssen wir in schwierigen Phasen besonders darauf achten, dass die Schüler*innen nicht noch stärker darunter leiden, sondern weiterhin bestmögliche Chancengerechtigkeit erreichen. 
Was stellt die Schule als Ort der Hoffnung jenen entgegen, die Zukunft als Katastrophe sehen?

Schule wird für viele Schüler*innen als Ort der Hoffnung wahrgenommen, wir müssen mit den Schüler*innen die Herausforderungen der Zukunft ansprechen, sie auch bestmöglich darauf vorbereiten. Das sind die Veränderungen der Berufswelt, aber auch die Klimathemen.

\section{Vielfalt als Selbstverständlichkeit}

Wir müssen die Schüler*innen auf die zahlreichen kulturellen Veränderungen vorbereiten. Auch wenn wir noch nicht wissen, was in den nächsten Jahrzehnten das Wichtigste ist, müssen wir unsere Schüler*innen umso flexibler und fitter machen. Das wird auch nicht nur im digitalen Bereich, im MINT-Bereich sein, sondern ganz besonders im sozialen Bereich notwendig werden. Gerade durch das verstärkte Aufkommen der Digitalen Schule müssen wir die Lehrer*innen auf die veränderte Unterrichtsgestaltung vorbereiten. Eben diese Veränderung, diese Vielfalt wird als Selbstverständlichkeit angenommen werden müssen.

\section{Welche Rolle spielt das Bildungsgeschehen insgesamt in diesen Entwicklungen?}

Bildung hat immer mit allem zu tun, es kommt in jedem Lebensabschnitt zum Tragen und bedeutet immer Veränderung so wie auch Entwicklung.

\section{Welche Verantwortung trägt jede einzelne Lehrperson darin?}

Jede Lehrkraft sollte wissen, dass Kinder vielfältige, unterschiedlich anregende Gelegenheiten benötigen, um ihre Chancen nutzen zu können. Lehrer*innen müssen die Herausforderungen der Änderungen der Unterrichtskultur erkennen. Die neue Lehr- und Lernkultur wird eine zentrale Anforderung für Lehrpersonen. Auch sie müssen in Zukunft fächerübergreifend, schulstufenübergreifend tätig werden. Sie werden viel mehr zu Lernbegleiter*innen und unterstützen damit die Schüler*innen. Sie werden sich auch viel mehr mit Umwelt- und Klimathemen beschäftigen. Die Lehrer*innen müssen auf die unterschiedlichen Ausgangslagen der Schüler*innen mehr denn je eingehen, manche Schüler*innen brauchen weniger, manche mehr Chancen, um an ihr Ziel zu kommen.

\section{Was ist die Herausforderung für die Schulaufsichtsbehörde angesichts dieser gesellschaftspolitischen Fragen, gerade und insbesondere im „Pädagogischen Dienst“ (wörtlich verstanden)?}

Für die Schulaufsichtsbehörde geht es angesichts dieser gesellschaftspolitischen Fragen vor allem um das Qualitätsmanagement und die strategische Entwicklung im Rahmen der Schulaufsicht sowie um die Errichtung von regionalen Schulaufsichtsteams in den Bildungsregionen. Die Bildungsaufsicht, insbesondere der Pädagogische Dienst, stellt und koordiniert sonder- und inklusionspädagogische Maßnahmen für Schüler*innen mit sonderpädagogischem und anderem Förderbedarf in allgemeinen Schulen und stellt für die Betreuung dieser Schüler*innen zusätzliche Lehrpersonen ein. 
Weiters wird am Bildungscontrolling mitgearbeitet, welches nach den Vorgaben der Geschäftsstelle für Qualitätsentwicklung und Qualitätssicherung und für die pädagogische Fachexpertise bei der Bewirtschaftung der Lehrpersonalressourcen zuständig ist. Für diese Aufgaben werden nach den regionalen Erfordernissen an den Außenstellen auch pädagogische Beratungszentren in der jeweiligen Bildungsdirektion implementiert.

Was heißt das für Sie, „HOFFNUNGsschule“? Was kennzeichnet eine solche?

Die Änderung der Kultur in der Schule kennzeichnet eine „HOFFNUNGsschule“. Lehr- und Lernprozesse werden sich in Zukunft ändern. Wir fragen uns ständig, welche Kompetenzen brauchen wir in Zukunft, wie können unsere Schüler*innen aktiv zusammenarbeiten, wodurch werden sie gerade besonders motivert, sich mit Themen der Zukunft auseinanderzusetzen, damit sie am Gesellschaftsleben glücklich und selbstbestimmt nach ihren Wünschen und Bedürfnissen teilhaben können und somit ihre persönlichen und zahlreiche Leuchttürme ansteuern und erreichen können.

Ich bedanke mich für das Gespräch und die Impulse zur Reflexion! Alles Gute, viel Kraft und Energie!

\section{Autorin}

Petra Heißenberger, HS-Prof. Mag. Dr. BEd MSc - Autorin

Petra Heißenberger ist Professorin für Schulmanagement und Leiterin des Zentrums Leadership an der Pädagogischen Hochschule Niederösterreich.

Kontakt: petra.heissenberger@ph-noe.ac.at 